(2) Open Access Full Text Article

REVIEW

\title{
Evaluating the Therapeutic Potential of Zanubrutinib in the Treatment of Relapsed/Refractory Mantle Cell Lymphoma: Evidence to Date
}

This article was published in the following Dove Press journal:

OncoTargets and Therapy

\section{Yazeed Sawalha $(\mathbb{D}$ \\ David A Bond (D) \\ Lapo Alinari}

Department of Internal Medicine, Division of Hematology, Arthur G. James Comprehensive Cancer Center, The Ohio State University Wexner Medical Center, Columbus, OH, USA
Correspondence: Yazeed Sawalha Department of Internal Medicine, Division of Hematology, The Ohio State University Comprehensive Cancer Center - Arthur G. James Cancer Hospital, A350B Starling-Loving Hall, 320 W. I0th Ave., Columbus, $\mathrm{OH} 43210$, USA $\mathrm{Tel}+\mid$ 614-293-0837

Fax + 614-293-7526

Email Yazeed.Sawalha@osumc.edu

\begin{abstract}
Mantle cell lymphoma (MCL) is an uncommon B-cell non-Hodgkin lymphoma characterized by an aggressive clinical course in the majority of patients. Despite recent improvements in outcomes, MCL remains incurable and a major therapeutic challenge. BTK inhibitors are the preferred treatment option for patients with relapsed/refractory MCL, including those unfit for chemotherapy or those with chemoresistant disease. In addition to ibrutinib and acalabrutinib, the FDA recently approved zanubrutinib for the treatment of patients with relapsed/refractory MCL based on the results of two Phase 2 clinical trials showing overall response rates of $85-87 \%$ with complete responses in $30-77 \%$ of patients. Compared with ibrutinib, zanubrutinib is more selective for BTK and has less off-target inhibition, which is thought to limit certain toxicities although direct comparative data are still lacking. This review article summarizes data from clinical trials of currently FDAapproved BTK inhibitors in MCL with a focus on zanubrutinib.
\end{abstract}

Keywords: BTK, zanubrutinib, ibrutinib, acalabrutinib

\section{Introduction}

Mantle cell lymphoma (MCL) is an uncommon subtype of B-cell non-Hodgkin lymphoma (NHL) that represents less than $10 \%$ of all NHL. ${ }^{1,2}$ MCL is characterized by translocation $(11 ; 14)(\mathrm{q} 13 ; \mathrm{q} 32)$, which results in cyclin D1 overexpression and cell cycle deregulation. Although cyclin D1 overexpression is the hallmark of MCL, it is insufficient for the development of MCL and the acquisition of other genetic alterations is required. ${ }^{3}$ The median age at diagnosis is 68 years with $3: 1$ male predilection. ${ }^{2}$ Two major subtypes of MCL are recognized based on molecular and clinical features. ${ }^{4}$ The classic MCL subtype is characterized by the presence of immunoglobulin heavy chain (IGHV) unmutated B cells with SOX11 expression and typically manifests with lymph node and extranodal involvement. The pleomorphic and blastoid forms are uncommon histologic variants of classic MCL and are usually associated with more aggressive presentation and poorer prognosis. The leukemic non-nodal MCL is a less common subtype characterized by the presence of IGHV mutated B cells without SOX11 expression, and typically involves the peripheral blood, bone marrow, and spleen. ${ }^{4}$ Risk stratification in MCL is based on clinical parameters included in the Mantle Cell Lymphoma Prognostic Index (MIPI) and histologic features such as the Ki-67 proliferation index. ${ }^{5,6}$

No unified treatment approach exists for patients with MCL. ${ }^{7}$ For the majority of patients, treatment is required at the time of diagnosis and selection of treatment 
is based on several factors including age, performance status, comorbidities, and patient/physician's preference. ${ }^{7}$ Younger fit patients are typically treated with intensive chemotherapy (generally defined as regimens including high-dose cytarabine) with or without consolidative autologous hematopoietic cell transplantation (HCT), ${ }^{8-12}$ whereas older or unfit patients are treated with lessintensive chemotherapy. ${ }^{13-16}$ Maintenance with rituximab is commonly considered in both approaches. ${ }^{12,13}$ Both intensive and less-intensive approaches result in high response rates that exceed $80 \%$ to $90 \%$, but intensive chemotherapy results in deeper responses and longer remissions. ${ }^{11}$ However, even in patients treated with intensive chemotherapy, relapses are inevitable with 4- to 6-year progression-free survival (PFS) of $50 \%$ to $65 \% .^{8-11}$

Relapsed MCL is a major therapeutic challenge. For fit patients who achieved durable responses with initial chemotherapy, retreatment with chemotherapy is often used but is usually less effective and results in shorter remissions. ${ }^{17}$ If not previously done, consolidative autologous HCT may be considered for fit patients with chemosensitive disease. ${ }^{18,19}$ In eligible patients, allogeneic HCT may lead to durable remissions but is associated with high treatment-related morbidity and mortality. ${ }^{19,20}$ There are six non-chemotherapy agents currently approved in the United States and/or Europe for the treatment of patients with relapsed/refractory MCL: bortezomib, temsirolimus, lenalidomide, and three Bruton's tyrosine kinase (BTK) inhibitors: ibrutinib, acalabrutinib, and zanubrutinib. Of these agents, the BTK inhibitors are generally considered the preferred treatment option for patients with relapsed/refractory MCL as they have the highest response rates and are generally well-tolerated. ${ }^{7}$ In this article, we review the role of BTK inhibitors in MCL with a focus on zanubrutinib.

\section{BTK Inhibitors in MCL}

BTK is a non-receptor kinase that belongs to the tyrosine protein kinase (Tec) family. Once recruited and activated by downstream signaling from the B-cell receptor (BCR), BTK's most important role is the activation of phospholipase C- $\gamma 2$ (PLC $\gamma 2$ ), which ultimately leads to the activation of several key pathways including nuclear factor- $\mathrm{\kappa B}$ (NF-kB), nuclear factor of activated T cells (NFAT), mitogen-activated protein kinase (MAPK), and mammalian target of rapamycin (AKT/mTOR) (Figure 1). ${ }^{21,22}$ In this way, BTK has a crucial role in amplifying signals from the BCR and is essential for B cell survival, maturation, differentiation, migration, and proliferation. ${ }^{23}$ The central role of BTK in B cell survival is evident in the X-linked agammaglobulinemia; a syndrome in which BTK loss-offunction mutations lead to the near absence of B cells and profound humoral immune deficiency. ${ }^{24}$ The importance of the BTK pathway is further highlighted by the success seen with the use of BTK inhibitors in several B-cell malignancies including MCL.

Three BTK inhibitors are currently approved by the US Food and Drug Administration (FDA) in relapsed/refractory MCL: ibrutinib, acalabrutinib, and zanubrutinib. These 3 BTK inhibitors inactivate BTK by irreversibly binding to a cysteine residue (C481S) of the adenosine triphosphate (ATP) binding-pocket in the kinase domain. Despite relatively short half-lives (acalabrutinib 1 hour, zanubrutinib 2-4 hours, ibrutinib 4-8 hours), the irreversible binding to C481S leads to reliable ( $>90 \%)$ and durable BTK inhibition after standard once- or twice-daily dosing. ${ }^{25-27}$ Other tyrosine kinases that contain a cysteine residue homologous to C481S within the ATP binding pocket may be susceptible to BTK inhibitors. These include TEC-family kinases such as TEC, interleukin-2inducible kinase (ITK), epidermal growth factor receptor (EGFR), human EGFR-2 (HER2), human EGFR-4 (HER4), and Janus Kinase 3 (JAK3). ${ }^{27}$ The off-target effects on other kinases explain, at least in part, some of the toxicities seen with BTK inhibitors such as rash and diarrhea (EGFR inhibition), bleeding (TEC inhibition), and atrial fibrillation (AF) (TEC inhibition). ${ }^{27,28}$ As discussed later in this article, the second-generation BTK inhibitors, acalabrutinib and zanubrutinib, were designed to have less off-target inhibition than ibrutinib and an enhanced safety profile. ${ }^{29}$ Beyond safety, the improved selectivity of the second-generation BTK inhibitors might impact their efficacy and potential for combination therapy. For example, due to its inhibition of ITK, a kinase that regulates the activation of $\mathrm{T}$ and natural killer cells, in vitro studies show that ibrutinib antagonizes the cellmediated cytotoxicity induced by anti-CD20 monoclonal antibodies, ${ }^{30-33}$ an effect not observed with acalabrutinib or zanubrutinib. ${ }^{33,34}$ These studies suggest that acalabrutinib and zanubrutinib may be better suited for combination treatment with anti-CD20 monoclonal antibodies, although this has not been confirmed in clinical trials.

Several other BTK inhibitors are currently in development for B-cell malignancies including MCL (Figure 1). Tirabrutinib (ONO-4059/GS-4059) and orelabrutinib (ICP-022) are alternative selective and irreversible BTK inhibitors that have shown promising activity in B-cell 


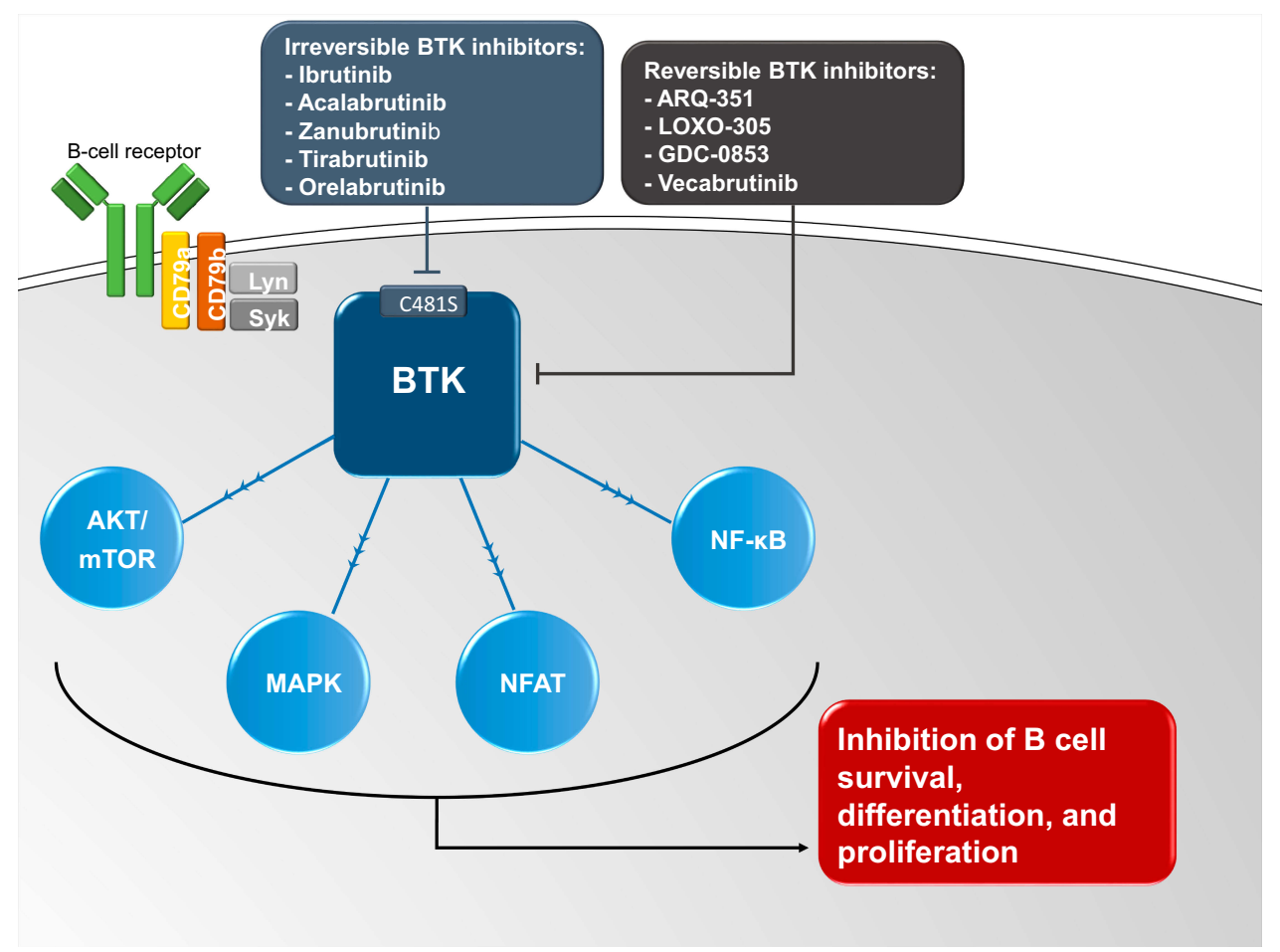

Figure I A simplified schematic of the role of Bruton's tyrosine kinase (BTK) in B cell receptor signaling and B cell survival. Ibrutinib, acalabrutinib, zanubrutinib, tirabrutinib, and orelabrutinib are irreversible BTK inhibitors that inactivate BTK by binding to C48IS. ARQ-35I, LOXO-305, GDC-0853, and vecabrutinib are reversible BTK inhibitors that inactivate BTK independent of C48IS.

Abbreviations: AKT/mTOR, mammalian target of rapamycin; LYN, Lck/Yes kinase; MAPK, mitogen-activated protein kinase; NFAT, nuclear factor of activated T cells; NF$\kappa B$, nuclear factor- $\kappa B$; SYK, spleen tyrosine kinase.

malignancies including MCL. ${ }^{35,36}$ ARQ-351, LOXO-305, GDC-0853, and vecabrutinib (SNS-062) are noncovalent reversible inhibitors of BTK that do not bind to C481S.

\section{Ibrutinib}

Ibrutinib was the first BTK inhibitor approved by the FDA in November 2013 for the treatment of patients with relapsed/refractory MCL. The recommended dose is $560 \mathrm{mg}$ orally once daily. In the pivotal phase 2 trial (PCYC-1104-CA) (Table 1), 111 patients with relapsed/ refractory MCL received ibrutinib $560 \mathrm{mg}$ daily until disease progression or unacceptable toxicity. ${ }^{37}$ Median age was 68 years (range, 40-84). The simplified MIPI score classified $38 \%$ and $49 \%$ of patients as having intermediateor high-risk disease, respectively. Patients were heavily pretreated with a median of 3 (range, 1-5) prior therapies, with $45 \%$ refractory to last therapy. Ibrutinib resulted in an objective response rate (ORR) of $68 \%$ including complete response (CR) in $21 \%$. With a median follow-up of 27 months, the median treatment duration was 8 months with $22 \%$ of patients treated for at least 2 years. ${ }^{38}$ The median duration of response (DOR), PFS, and overall survival
(OS) were 18, 13, and 23 months, respectively. The most common adverse events (AEs) of any grade included diarrhea $(54 \%)$, fatigue $(50 \%)$, nausea (33\%), and dyspnea (32\%). Grade 3-4 AEs included neutropenia (17\%), anemia $(11 \%)$, pneumonia $(6 \%)$, and bleeding $(6 \%)$. Four patients had a subdural hematoma. AEs led to treatment discontinuation in $11 \%$ of patients. ${ }^{37,38}$ A Phase 3 trial of ibrutinib vs temsirolimus (MCL3001) in 280 patients with relapsed/refractory MCL showed similar response rates with ibrutinib (Table 1). ${ }^{39,40}$ In this trial, the median age was 68 years, the median number of prior therapies was 2 (range, 1-9), and $30 \%$ of patients were refractory to last therapy. Patients had intermediate- or high-risk disease as classified by the simplified MIPI score in $48 \%$ and $21 \%$, respectively. Ibrutinib resulted in $77 \%$ ORR including $23 \%$ CR compared with $47 \%$ ORR including 3\% CR with temsirolimus. With a median follow-up of 39 months, the median DOR, PFS, and OS were longer with ibrutinib compared with temsirolimus $(23,16$, and 30 vs 6,6 , and 24 months, respectively). In the ibrutinib arm, common toxicities of any grade included diarrhea (33\%), fatigue (24\%), and cough (23\%). AEs of interest in the ibrutinib 


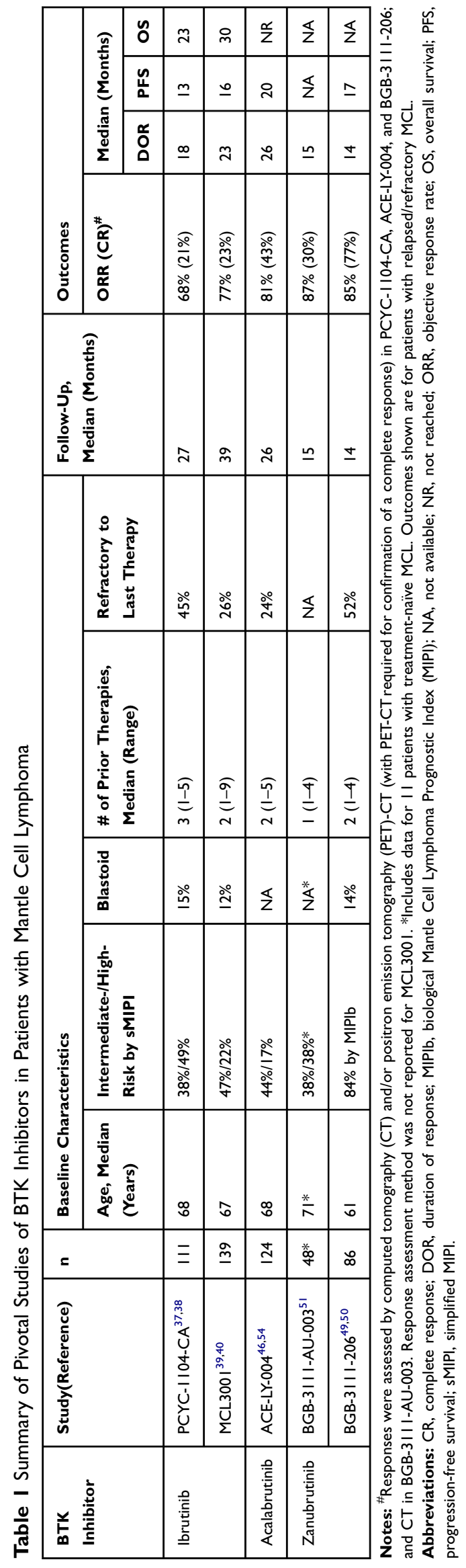

arm included major bleeding in $9 \%$ and grade $\geq 3 \mathrm{AF}$ in $5 \%$ of patients. AEs led to ibrutinib discontinuation in $17 \%$ of patients. ${ }^{39,40}$

A pooled analysis reported on the outcomes and safety data of 370 patients with relapsed/refractory MCL treated with ibrutinib in 3 clinical trials (PCYC-1104-CA, MCL3001, and MCL2001) with a median follow-up of 41 months. ${ }^{41-43}$ Median treatment duration with ibrutinib was 11 months with $22 \%$ of patients remaining on ibrutinib for at least 3 years. Median age was 68 years and median number of prior therapies was 2 (range, 1-9). Compared with patients with $\geq 2$ prior therapies, those with 1 prior therapy had significantly longer median DOR (36 vs 17 months), PFS ( 25 vs 10 months), and OS (62 vs 23 months). Common grade $\geq 3$ AEs were neutropenia $(17 \%)$, thrombocytopenia (12\%), pneumonia (13\%), anemia (10\%), $\mathrm{AF} /$ atrial flutter $(6 \%)$, and hypertension (5\%). Any-grade AF was reported in $11 \%$ of the patients and grade $\geq 3$ bleeding was reported in $5 \%$. AEs led to treatment discontinuation in $10 \%$ of patients. ${ }^{41,42}$ Another pooled analysis reported on the safety data of ibrutinib in 4 randomized clinical trials of patients with chronic lymphocytic leukemia (CLL)/small lymphocytic lymphoma (RESONATE, RESONATE-2, HELIOS) or MCL (MCL3001). ${ }^{44}$ The analysis included 756 patients treated with ibrutinib (469 as a single agent and 287 in combination with bendamustine plus rituximab) and 749 patients treated with comparators. For both cohorts, the median age was 67 years (range, 30-90) and the median number of prior therapies was 2 (range, $0-13$ ). The median treatment duration was 13 months (range, 0-28) for ibrutinib and 6 months (range, 0-27) for comparators. The most common AEs of any grade (in $\geq 20 \%$ of patients) with ibrutinib were diarrhea (39\%), neutropenia (33\%), nausea (27\%), fatigue $(25 \%)$, pyrexia (22\%), thrombocytopenia $(21 \%)$, and anemia (21\%). Of the AEs occurring in $\geq 10 \%$ of patients, only diarrhea, arthralgia (13\%), and muscle spasms (13\%) were more common with ibrutinib than with the comparators when adjusted for exposure. Common grade $\geq 3$ AEs (in $\geq 3 \%$ of patients) with ibrutinib included neutropenia (29\%), thrombocytopenia (9\%), pneumonia $(8 \%)$, febrile neutropenia $(6 \%)$, anemia (5\%), hypertension $(3 \%)$, AF $(3 \%)$, diarrhea (3\%), and fatigue (3\%). Grade $\geq 3$ bleeding was reported in $3 \%$ of ibrutinib-treated patients. Diarrhea, hypertension, and AF were the only grade $\geq 3$ AEs more common with ibrutinib than with the comparators when adjusted for exposure. AEs led to treatment discontinuation in $12 \%$ of ibrutinib-treated patients. ${ }^{44}$ 


\section{Acalabrutinib}

Compared with ibrutinib, acalabrutinib has improved pharmacologic features with shorter half-life, rapid oral absorption, and absence of irreversible targeting of alternative kinases such as EGFR, TEC, and ITK. ${ }^{29,45}$ The FDA approved acalabrutinib for the treatment of patients with relapsed/refractory MCL in October 2017. The recommended dose is $100 \mathrm{mg}$ orally every 12 hours. In the pivotal phase 2 trial (ACE-LY-004), 124 patients with relapsed/ refractory MCL were treated with acalabrutinib $100 \mathrm{mg}$ every 12 hours until disease progression or unacceptable toxicity (Table 1). ${ }^{46,47}$ Median age was 68 years (range, $42-90)$. By the simplified MIPI risk score, $44 \%$ and $17 \%$ of patients had high intermediate- and high-risk disease, respectively. The median number of prior therapies was 2 (range, $1-5$ ) with $24 \%$ of patients being refractory to last therapy. The trial results were updated with a median follow-up of 26 months. ${ }^{47}$ Acalabrutinib resulted in an ORR of $81 \%$ including CR in 43\%. The median DOR and PFS were 26 and 20 months, respectively, whereas the median OS was not reached. ${ }^{46,47}$ The most common AEs of any grade were headache $(38 \%)$, diarrhea (36\%), fatigue $(28 \%)$, cough $(22 \%)$, and myalgia (21\%). Headache was mostly grade 1 $(64 \%)$, occurred as one event in the majority of patients (77\%), and lasted for a median of 11 days. Grade $\geq 3$ AEs included anemia (10\%), neutropenia (10\%), and pneumonia $(6 \%)$. One case of AF was reported. Bleeding events occurred in $33 \%$ of patients and were grade $1-2$ except 3 grade 3 bleeding events (gastrointestinal bleeding, hematuria, hematoma). AEs led to treatment discontinuation in $8 \%$ of patients. ${ }^{46,47}$

A pooled analysis of 7 clinical trials of acalabrutinib monotherapy in 610 patients with various B-cell malignancies including MCL, CLL, diffuse large B-cell lymphoma, follicular lymphoma, Waldenström macroglobulinemia (WM) and multiple myeloma reported on the safety data of acalabrutinib. ${ }^{48}$ The median age of this patient population was 66 years (range, 32-90), and the median number of prior therapies was 1 (range, $0-13$ ). The median duration of treatment with acalabrutinib was 14 months (range, 0-32). The most common AEs of any grade (in $\geq 20 \%$ of patients) were headache $(42 \%)$, diarrhea $(38 \%)$, fatigue $(23 \%)$, nausea $(23 \%)$, and contusion $(22 \%)$. The most common grade $\geq 3$ AEs (in $\geq 3 \%$ of patients) were neutropenia (9\%), anemia (7\%), pneumonia (6\%), and thrombocytopenia (4\%). Grade 5 AEs occurred in 22 patients ( $4 \%$ ) and included pneumonia $(n=6,3$ deemed treatment-related), liver failure due to hepatitis $\mathrm{B}$ reactivation $(\mathrm{n}=1)$, and intracranial hematoma $(n=1)$. Serious or grade $\geq 3$ bleeding was reported in 15 patients $(3 \%)$, most frequently in the gastrointestinal tract $(n=5)$ and central nervous system $(n=3)$. AF of any grade was reported in $2 \%$ (grade 3 in $1 \%$ ). AEs led to treatment discontinuation in $6 \%$ of patients. ${ }^{48}$

\section{Zanubrutinib}

Similar to acalabrutinib, zanubrutinib (formerly BGB-3111) also showed greater BTK selectivity and less off-target inhibition against alternative kinases including EGFR, ITK, HER2, and TEC compared with ibrutinib. ${ }^{26,29}$ Zanubrutinib was approved by the FDA in November 2019 for the treatment of patients with MCL who had received at least one prior therapy based on the results of two studies: BGB-3111AU-003 and BGB-3111-206. ${ }^{26,49,50}$ The recommended dose is $160 \mathrm{mg}$ orally twice daily or $320 \mathrm{mg}$ orally once daily. BGB-3111-AU-003 was an international first-in-human Phase 1 clinical trial of zanubrutinib comprising a total of 144 patients with various B-cell malignancies. Seventeen patients were enrolled in part 1 (dose-escalation cohort), 39 in cohort $2 \mathrm{a}$ (dose-finding cohort), and 94 patients with CLL/ small lymphocytic lymphoma were enrolled in part $2 .^{26}$ Patients received zanubrutinib at escalating dose levels ranging from $40 \mathrm{mg}$ to $320 \mathrm{mg}$ daily (the $320 \mathrm{mg}$ dose was given as either a single dose or $160 \mathrm{mg}$ twice daily) until disease progression or unacceptable toxicity. Based on pharmacokinetic and pharmacodynamic data as well as toxicity assessment (no dose-limiting toxicities were observed), both the $320 \mathrm{mg}$ daily dose and the $160 \mathrm{mg}$ twice daily dose were selected for further evaluation. An enzyme-linked immunosorbent assay-based evaluation of BTK occupancy was performed in peripheral blood mononuclear cells (PBMCs) from 45 patients across all cohorts and in paired lymph node biopsy specimens from 30 patients. Greater than 95\% BTK occupancy at 4 hours post zanubrutinib was achieved in the PBMCs of almost all patients with no significant differences across dose groups. In lymph nodes, BTK occupancy (predose on day 3 of week 1) was significantly higher with $160 \mathrm{mg}$ twice-daily dosage (median $=100 \%$, range $86 \%$ $100 \%$; $>95 \%$ occupancy in $89 \%$ of patients) than with $320 \mathrm{mg}$ once-daily dosage (median $=94 \%$, range $82 \%$ $100 \% ;>95 \%$ occupancy in $50 \%$ of patients). Based on the higher nodal BTK occupancy, the $160 \mathrm{mg}$ twice-daily dose was selected as the recommended phase 2 dose (patients already being treated on the $320 \mathrm{mg}$ daily dose were encouraged to switch to $160 \mathrm{mg}$ twice daily). Whether the higher nodal BTK occupancy achieved with twice-daily dosing 
impacts clinical outcomes is unclear and both dosing schedules are recommended in the FDA label for zanubrutinib. The most common AEs occurring with zanubrutinib in patients treated on part 1 and cohort $2 \mathrm{a}(\mathrm{n}=56)$ were upper respiratory tract infection (39\%), contusion (36\%), cough (27\%), and diarrhea (27\%). Grade $\geq 3$ AEs reported in $>2$ patients were anemia $(13 \%)$, pneumonia $(7 \%)$, pyrexia $(7 \%)$, acute kidney injury (5\%), and neutropenia (5\%). AEs led to treatment discontinuation in $14 \%$ of patients. No significant differences in AEs were seen between the $320 \mathrm{mg}$ daily and 160 twice daily dosing schedules, although the number of patients in each group was small (19 and 25 patients, respectively). ${ }^{26}$

The MCL expansion cohort of BGB-3111-AU-003 comprised 48 patients, including 11 patients with previously untreated MCL and 37 with relapsed/refractory MCL (Table 1). Patients received zanubrutinib $160 \mathrm{mg}$ twice daily or $320 \mathrm{mg}$ daily until disease progression or unacceptable toxicity. Updated results were presented at the 15 th International Conference on Malignant Lymphoma in 2019. ${ }^{51}$ Median age was 71 (range, 42-90). The MIPI score indicated intermediate- and high-risk disease each in $38 \%$ of patients. The median number of prior therapies for patients with relapsed/refractory MCL was 1 (range, 1-4). The ORR and CR rate were $88 \%$ and $38 \%$ in previously untreated patients, and $87 \%$ and $30 \%$ in those with relapsed/ refractory MCL. With a median follow-up of 15 months in patients with previously untreated MCL and 14 months in those with relapsed/refractory MCL, the median DOR was 15 months in both groups. The most common AEs included diarrhea (35\%), petechiae/purpura/contusion (31\%), upper respiratory tract infection $(27 \%)$, rash $(19 \%)$, headache (17\%) and peripheral edema (17\%). Ten patients $(21 \%)$ discontinued treatment due to AEs (pneumonia $(n=2)$, subdural hematoma, renal hematoma, peripheral edema, heart failure, thalamic infarction, lung cancer, myelodysplastic syndrome, vasculitis). Five patients died due to AEs (2 sepsis, 1 pneumonia, 1 heart failure, 1 thalamic infarction) but none were deemed related to zanubrutinib.

In the phase 2 BGB-3111-206, 86 patients with relapsed/ refractory MCL were treated with zanubrutinib $160 \mathrm{mg}$ twice daily until disease progression or unacceptable toxicity at 13 centers in China (Table 1). ${ }^{49,50}$ Median age was 61 years (range, $34-75$ ), and $84 \%$ of patients had intermediateor high-risk disease by the MIPIb score. The median number of prior therapies was 2 (range, $1-4$ ) with $52 \%$ of patients refractory to last therapy. Zanubrutinib resulted in an ORR of $85 \%$ including $\mathrm{CR}$ in $77 \%$. With a median follow-up of
14 months, the median DOR and PFS were 14 and 17 months, respectively. Grade $\geq 3$ AEs included neutropenia (16\%), decreased WBC count (6\%), anemia (6\%), lung infection (6\%), thrombocytopenia (5\%), and hypertension (4\%). No cases of AF were reported. The most frequent bleeding events were hematuria and petechiae/purpura/contusion (5\% each, grade $1-2)$ whereas serious or grade $\geq 3$ bleeding was reported in 2 patients $(2 \%)$. AEs led to treatment discontinuation in $11 \%$ of patients. ${ }^{49,50}$ The type of imaging modality used for response assessment might explain the discrepancy between the $\mathrm{CR}$ rates in BGB3111-206 (positron emission tomography) and BGB-3111AU-003 (computed tomography).

Focusing further on the safety profile of zanubrutinib, an analysis of pooled data from six ongoing clinical trials reported the safety of zanubrutinib $160 \mathrm{mg}$ twice daily or $320 \mathrm{mg}$ daily in 671 patients with B-cell malignancies. ${ }^{52}$ Almost half of the patients $(46 \%)$ were treated on studies conducted in China. The median age was 64 years (range, 20-90), and the median duration of treatment with zanubrutinib was 11 months (range, 0.1-47). The most common AEs included diarrhea (18\%), cough (19\%), contusion (19\%), and rash (18\%). Grade $\geq 3$ AEs reported in $\geq 3 \%$ were neutropenia $(14 \%)$, anemia $(8 \%)$, thrombocytopenia $(4 \%)$, pneumonia (5\%), lung infection (4\%), and hypertension $(3 \%)$, with the most common serious AEs being pneumonia (5\%) and lung infection (3\%). Bleeding occurred in $46 \%$ of patients including grade $\geq 3$ in $2 \%$. The most common bleeding events were contusion (17\%) and hematuria (11\%). Major hemorrhage, defined as serious or grade $\geq 3$ bleeding at any site, or central nervous system bleeding of any grade, occurred in 3\%. The median time to any bleeding event was 63 days (range, 3-601). AF developed in 2\% of patients. Infections occurred in $67 \%$ of patients including grade $\geq 3$ in $21 \%$. Diarrhea was grade $\geq 3$ in $1 \%$. Hypertension was reported in $8 \%$ including grade $\geq 3$ in $3 \%$. AEs resulting in death in more than one patient were pneumonia $(n=6)$, septic shock $(n=2)$, multiple organ failure $(n=2)$, and unspecified $(n=5)$. Treatment with zanubrutinib was discontinued in $10 \%$ of patients due to AEs, including $5 \%$ due to treatment-related AEs, with a median time to treatment discontinuation of 3.5 months (range, 0.1-41). Treatment-related AEs leading to zanubrutinib discontinuation in $>1$ patient were pneumonia $(n=4)$, lung infection $(n=4)$, and thrombocytopenia $(n=2)$. Zanubrutinib dose reduction was required in $4 \%$ of patients. ${ }^{52}$

Table 2 lists the incidence of selected AEs reported with ibrutinib, acalabrutinib, and zanubrutinib. Caution 
Table 2 Adverse Events Reported with Ibrutinib, Acalabrutinib, and Zanubrutinib

\begin{tabular}{|c|c|c|c|}
\hline Adverse Events (AEs) & Ibrutinib $(n=370)^{41-43}$ & Acalabrutinib* $(n=6 \mid 0)^{48}$ & Zanubrutinib* $(n=67 I)^{52}$ \\
\hline Headache, any grade (grade $\geq 3$ ) & $37 \%(0 \%)$ & $42 \%(2 \%)$ & $4 \%$ (not reported) $* *$ \\
\hline Diarrhea, any grade (grade $\geq 3$ ) & $40 \%(4 \%)$ & $38 \%(2 \%)$ & $18 \%(1 \%)$ \\
\hline Hypertension, grade $\geq 3$ & $5 \%$ & $<3 \%$ & $3 \%$ \\
\hline Atrial fibrillation, any grade (grade $\geq 3$ ) & $11 \%(6 \%)$ & $2 \%(1 \%)$ & $2 \%(1 \%)$ \\
\hline Bleeding, serious or grade $\geq 3$ & $5 \%$ & $3 \%$ & $3 \%$ \\
\hline Neutropenia, grade $\geq 3$ & $17 \%$ & $9 \%$ & $14 \%$ \\
\hline Thrombocytopenia, grade $\geq 3$ & $12 \%$ & $4 \%$ & $4 \%$ \\
\hline Anemia, grade $\geq 3$ & $10 \%$ & $7 \%$ & $8 \%$ \\
\hline Treatment discontinuation due to AEs & $10 \%$ & $6 \%$ & $10 \%$ \\
\hline
\end{tabular}

Notes: *Studies included patients with MCL and other B-cell malignancies. **From zanubrutinib's package insert.

should be exercised when comparing results across studies, noting differences in the enrolled patient populations and in particular that patients in the ibrutinib studies were more heavily pretreated (Table 1), which has been associated with higher rates of AEs. ${ }^{42}$ In addition, due to the relatively short follow-up duration of patients treated with zanubrutinib in these studies, late or cumulative toxicities of zanubrutinib may be underreported. Notable differences in AEs among these 3 BTK inhibitors include lower incidence of AF with acalabrutinib and zanubrutinib, and lower incidence of headache and diarrhea with zanubrutinib. AEs led to treatment discontinuation in $10 \%$ of patients treated with ibrutinib or zanubrutinib and in $6 \%$ of those treated with acalabrutinib. Data from the first clinical trial to directly compare 2 BTK inhibitors, the ASPEN trial, was recently presented at the American Society of Clinical Oncology 2020 Annual Meeting. ${ }^{53}$ In this phase 3 clinical trial, 201 patients with $\mathrm{WM}$ were randomized to ibrutinib $420 \mathrm{mg}$ daily or zanubrutinib $160 \mathrm{mg}$ twice daily until disease progression. Although the trial did not meet its primary end-point (CR and very good PR rates with zanubrutinib were not superior to those achieved with ibrutinib), it highlighted important differences in the safety profiles of ibrutinib and zanubrutinib. Compared with ibrutinib, zanubrutinib resulted in lower rates of diarrhea ( $22 \%$ vs $32 \%$ ), contusion ( $13 \%$ vs $24 \%$ ), muscle spasms $(10 \%$ vs $24 \%)$, peripheral edema ( $9 \%$ vs $19 \%$ ), grade $\geq 3$ hypertension ( $8 \%$ vs $15 \%$ ), major hemorrhage ( $6 \%$ vs $10 \%$ ), AF/atrial flutter ( $3 \%$ vs $18 \%$, with no cases of grade $\geq 3 \mathrm{AF}$ with zanubrutinib compared with $7 \%$ with ibrutinib), and grade $\geq 3$ pneumonia ( $1 \%$ vs $7 \%$ ). Zanubrutinib resulted in higher rate of grade $\geq 3$ neutropenia $(22 \%$ vs $8 \%)$ without increased rate of infection $(19 \%$ vs $24 \%$ ). Further, zanubrutinib resulted in fewer AEs leading to dose reductions ( $14 \%$ vs $24 \%)$, treatment interruption $(47 \%$ vs $56 \%)$ or discontinuation ( $4 \%$ vs $14 \%)$, and death ( $1 \%$ vs $4 \%)$. The ASPEN trial shows that zanubrutinib has a better overall safety profile than ibrutinib. ${ }^{53}$ The ongoing phase 3 ALPINE clinical trial (NCT03734016) comparing zanubrutinib with ibrutinib in patients with CLL will be important to confirm these results. In addition, ELEVATE-RR (NCT02477696) is an ongoing phase 3 clinical trial comparing acalabrutinib with ibrutinib in patients with CLL. Currently, either acalabrutinib or zanubrutinib may be the preferred option in patients at increased risk for cardiovascular or bleeding events. Ibrutinib and zanubrutinib may be preferred over acalabrutinib in patients at risk for medication nonadherence with a twice-daily dosing schedule.

\section{Conclusion and Future Directions}

Zanubrutinib is now the third irreversible BTK inhibitor approved for patients with relapsed/refractory MCL. Crosstrial comparisons show similar efficacy for ibrutinib, acalabrutinib, and zanubrutinib in relapsed/refractory MCL. However, the toxicity profiles of these BTK inhibitors appear to vary, and the more selective BTK inhibitors zanubrutinib and acalabrutinib may be associated with a lower incidence of some toxicities of interest including cardiovascular toxicity. Results of ongoing randomized clinical trials comparing acalabrutinib and zanubrutinib with ibrutinib will be important in differentiating the safety profile of each BTK inhibitor and guiding treatment selection. As a selective BTK inhibitor, zanubrutinib is an attractive option to combine with other targeted agents in B-cell malignancies including MCL. Ongoing clinical trials of such combinations include zanubrutinib plus ME-401 (a phosphatidylinositol-3-kinase inhibitor) (NCT02914938) and zanubrutinib plus tislelizumab (anti-PD-1 monoclonal antibody) (NCT02795182). NCT04002297 is a phase 3 
clinical trial comparing zanubrutinib plus rituximab with bendamustine plus rituximab in patients with previously untreated MCL who are not eligible for autologous HCT.

\section{Acknowledgment}

The authors would like to thank Dr. Kami Maddocks (The Ohio State University) for her insightful review and comments on this article.

\section{Disclosure}

The authors report no conflicts of interest in this work.

\section{References}

1. Al-Hamadani M, Habermann TM, Cerhan JR, Macon WR, Maurer MJ, Go RS. Non-Hodgkin lymphoma subtype distribution, geodemographic patterns, and survival in the US: a longitudinal analysis of the national cancer data base from 1998 to 2011. Am J Hematol.. 2015;90(9):790-795. doi:10.1002/ajh.24086

2. Zhou Y, Wang H, Fang W, et al. Incidence trends of mantle cell lymphoma in the United States between 1992 and 2004. Cancer. 2008;113(4):791-798. doi:10.1002/cncr.23608

3. Jares P, Colomer D, Campo E, Jares P, Colomer D, Campo E. Molecular pathogenesis of mantle cell lymphoma. Find the latest version: review series Molecular pathogenesis of mantle cell lymphoma. J Clin Investig. 2012;122(10):3416-3423. doi:10.1172/ JCI61272.3416

4. Swerdlow SH, Campo E, Pileri SA, et al. The 2016 revision of the World Health Organization classification of lymphoid neoplasms. Blood. 2016;127(20):2375-2390. doi:10.1182/blood-2016-01-643569

5. Hoster E, Dreyling M, Klapper W, et al. A new prognostic index (MIPI) for patients with advanced-stage mantle cell lymphoma. Blood. 2008;111(2):558-565. doi:10.1182/blood-2007-06-095331

6. Hoster E, Rosenwald A, Berger F, et al. Prognostic value of Ki-67 index, cytology, and growth pattern in mantle-cell lymphoma: results from randomized trials of the european mantle cell lymphoma network. J Clin Oncol. 2016;34(12):1386-1394. doi:10.1200/ JCO.2015.63.8387

7. Maddocks K. Update on mantle cell lymphoma. Blood. 2018;132 (16):1647-1656. doi:10.1182/blood-2018-03-791392

8. Merli F, Luminari S, Ilariucci F, et al. Rituximab plus HyperCVAD alternating with high dose cytarabine and methotrexate for the initial treatment of patients with mantle cell lymphoma, a multicentre trial from Gruppo Italiano Studio Linfomi. Br J Haematol. 2012;156 (3):346-353. doi:10.1111/j.1365-2141.2011.08958.x

9. Chihara D, Cheah CY, Westin JR, et al. Rituximab plus hyper-CVAD alternating with MTX/Ara-C in patients with newly diagnosed mantle cell lymphoma: 15-year follow-up of a Phase II study from the MD Anderson Cancer Center. Br J Haematol. 2016;172(1):80-88. doi:10.1111/bjh.13796

10. Eskelund CW, Kolstad A, Jerkeman M, et al. 15-year follow-up of the Second Nordic Mantle Cell Lymphoma trial (MCL2): prolonged remissions without survival plateau. Br J Haematol. 2016;175 (3):410-418. doi:10.1111/bjh.14241

11. Hermine O, Hoster E, Walewski J, et al. Addition of high-dose cytarabine to immunochemotherapy before autologous stem-cell transplantation in patients aged 65 years or younger with mantle cell lymphoma (MCL Younger): a randomised, open-label, phase 3 trial of the European Mantle Cell Lymphoma N. Lancet. 2016;388 (10044):565-575. doi:10.1016/S0140-6736(16)00739-X
12. Le Gouill S, Thieblemont C, Oberic L, et al. Rituximab after autologous stem-cell transplantation in mantle-cell lymphoma. $N$ Engl $J$ Med. 2017;377(13):1250-1260. doi:10.1056/NEJMoa1701769

13. Kluin-Nelemans HC, Hoster E, Hermine O, et al. Treatment of older patients with mantle-cell lymphoma. $N$ Engl J Med. 2012;367 (6):520-531. doi:10.1056/NEJMoa1200920

14. Rummel MJ, Niederle N, Maschmeyer G, et al. Bendamustine plus rituximab versus CHOP plus rituximab as first-line treatment for patients with indolent and mantle-cell lymphomas: an open-label, multicentre, randomised, phase 3 non-inferiority trial. Lancet. 2013;381(9873):1203-1210. doi:10.1016/S0140-6736(12)61763-2

15. Flinn IW, Van Der Jagt R, Kahl BS, et al. Randomized trial of bendamustine-rituximab or R-CHOP/R-CVP in first-line treatment of indolent NHL or MCL: the BRIGHT study. Blood. 2014;123 (19):2944-2952. doi:10.1182/blood-2013-11-531327

16. Robak T, Huang H, Jin J, et al. Bortezomib-based therapy for newly diagnosed mantle-cell lymphoma. $N$ Engl $J$ Med. 2015;372 (10):944-953. doi:10.1056/NEJMoa1412096

17. Kumar A, Sha F, Toure A, et al. Patterns of survival in patients with recurrent mantle cell lymphoma in the modern era: progressive shortening in response duration and survival after each relapse. Blood Cancer J. 2019;9(6). doi:10.1038/s41408-019-0209-5

18. Till BG, Gooley TA, Crawford N, et al. Effect of remission status and induction chemotherapy regimen on outcome of autologous stem cell transplantation for mantle cell lymphoma. Leuk Lymphoma. 2008;49 (6):1062-1073. doi:10.1080/10428190801923725

19. Fenske TS, Zhang MJ, Carreras J, et al. Autologous or reduced-intensity conditioning allogeneic hematopoietic cell transplantation for chemotherapy-sensitive mantle-cell lymphoma: analysis of transplantation timing and modality. J Clin Oncol. 2014;32 (4):273-281. doi:10.1200/JCO.2013.49.2454

20. Lin RJ, Ho C, Hilden PD, et al. Allogeneic haematopoietic cell transplantation impacts on outcomes of mantle cell lymphoma with TP 53 alterations. Br J Haematol. 2018:bjh.15721. doi:10.1111/ bjh. 15721 .

21. Merolle MI, Ahmed M, Nomie K, Wang ML. The B cell receptor signaling pathway in mantle cell lymphoma. Oncotarget. 2018;9 (38):25332-25341. doi:10.18632/oncotarget.25011

22. Hendriks RW, Yuvaraj S, Kil LP. Targeting Bruton's tyrosine kinase in B cell malignancies. Nat Rev Cancer. 2014;14(4):219-232. doi: $10.1038 / \mathrm{nrc} 3702$

23. Niiro H, Clark EA. Regulation of B-cell fate by antigen-receptor signals. Nat Rev Immunol. 2002;2(12):945-956. doi:10.1038/nri955

24. Tsukada S, Saffran DC, Rawlings DJ, et al. Deficient expression of a B cell cytoplasmic tyrosine kinase in human X-linked agammaglobulinemia. Cell. 1993;72(2):279-290. doi:10.1016/00928674(93)90667-f

25. Advani RH, Buggy JJ, Sharman JP, et al. Bruton tyrosine kinase inhibitor ibrutinib (PCI-32765) has significant activity in patients with relapsed/refractory B-cell malignancies. J Clin Oncol. 2013;31 (1):88-94. doi:10.1200/JCO.2012.42.7906

26. Tam CS, Trotman J, Opat S, et al. Phase 1 study of the selective BTK inhibitor zanubrutinib in B-cell malignancies and safety and efficacy evaluation in CLL. Blood. 2019;134(11):851-859. doi:10.1182/ blood.2019001160

27. Byrd JC, Harrington B, O'Brien S, et al. Acalabrutinib (ACP-196) in relapsed chronic lymphocytic leukemia. $N$ Engl J Med. 2016;374 (4):323-332. doi:10.1056/NEJMoa1509981

28. McMullen JR, Boey EJH, Ooi JYY, Seymour JF, Keating MJ, Tam CS. Ibrutinib increases the risk of atrial fibrillation, potentially through inhibition of cardiac PI3K-Akt signaling. Blood. 2014;124 (25):3829-3830. doi:10.1182/blood-2014-10-604272

29. Wu J, Liu C, Tsui ST, Liu D. Second-generation inhibitors of Bruton tyrosine kinase. J Hematol Oncol. 2016;9(1):42-46. doi:10.1186/ s13045-016-0313-y 
30. Khurana D, Arneson LN, Schoon RA, Dick CJ, Leibson PJ. Differential regulation of human NK cell-mediated cytotoxicity by the tyrosine kinase Itk. J Immunol. 2007;178(6):3575-3582. doi:10. 4049/jimmunol.178.6.3575

31. Da Roit F, Engelberts PJ, Taylor RP, et al. Ibrutinib interferes with the cell-mediated anti-tumor activities of therapeutic CD20 antibodies: implications for combination therapy. Haematologica. 2015;100 (1):77-86. doi:10.3324/haematol.2014.107011

32. Kohrt HE, Sagiv-Barfi I, Rafiq S, et al. Ibrutinib antagonizes rituximab-dependent NK cell-mediated cytotoxicity. Blood. 2014;123(12):1957-1960. doi:10.1182/blood-2014-01-547869

33. Rajasekaran N, Sadaram M, Hebb J, et al. Three BTK-specific inhibitors, in contrast to ibrutinib, do not antagonize rituximab-dependent NK-cell mediated cytotoxicity. Blood. 2014;124(21):3118. doi:10. 1182/blood.V124.21.3118.3118

34. Flinsenberg TWH, Tromedjo CC, Hu N, et al. Differential effects of BTK inhibitors ibrutinib and zanubrutinib on NK-cell effector function in patients with mantle cell lymphoma. Haematologica. 2020;105(2):e76-e79. doi:10.3324/haematol.2019.220590

35. Munakata W, Ando K, Hatake K, et al. Phase I study of tirabrutinib (ONO-4059/GS-4059) in patients with relapsed or refractory B-cell malignancies in Japan. Cancer Sci. 2019;110(5):1686-1694. doi:10.1111/cas. 13983

36. Song Y, Song Y, Liu L, et al. Safety and efficacy of orelabrutinib monotherapy in Chinese patients with relapsed or refractory mantle cell lymphoma: a multicenter, open-label, phase II study. Blood. 2019;134(Supplement_1):755. doi:10.1182/blood-2019-126305

37. Wang ML, Rule S, Martin P, et al. Targeting BTK with ibrutinib in relapsed or refractory mantle-cell lymphoma. $N$ Engl J Med. 2013;369(6):507-516. doi:10.1056/NEJMoa1306220

38. Wang ML, Blum KA, Martin P, et al. Long-term follow-up of MCL patients treated with single-agent ibrutinib: updated safety and efficacy results. Blood. 2015;126(6):739-745. doi:10.1182/blood-201503-635326

39. Dreyling M, Jurczak W, Jerkeman M, et al. Ibrutinib versus temsirolimus in patients with relapsed or refractory mantle-cell lymphoma: an international, randomised, open-label, phase 3 study. Lancet 2016;387(10020):770-778. doi:10.1016/S0140-6736(15)00667-4

40. Rule S, Jurczak W, Jerkeman M, et al. Ibrutinib versus temsirolimus: 3-year follow-up of patients with previously treated mantle cell lymphoma from the phase 3, international, randomized, open-label RAY study. Leukemia. 2018;32(8):1799-1803. doi:10.1038/s41375018-0023-2

41. Rule S, Dreyling M, Goy A, et al. Outcomes in 370 patients with mantle cell lymphoma treated with ibrutinib: a pooled analysis from three open-label studies. Br J Haematol. 2017;179(3):430-438. doi:10.1111/bjh.14870

42. Rule S, Dreyling M, Goy A, et al. Ibrutinib for the treatment of relapsed/refractory mantle cell lymphoma: extended 3.5-year follow up from a pooled analysis. Haematologica. 2019;104(5):e211-e214. doi:10.3324/haematol.2018.205229
43. Rule S, Dreyling MH, Goy A, et al. Long-term outcomes with ibrutinib versus the prior regimen: a pooled analysis in relapsed/ refractory $(\mathrm{R} / \mathrm{R})$ Mantle Cell Lymphoma (MCL) with up to 7.5 years of extended follow-up. Blood. 2019;134(Supplement_1):1538. doi:10.1182/blood-2019-124691

44. O'Brien S, Hillmen P, Coutre S, et al. Safety analysis of four randomized controlled studies of ibrutinib in patients with chronic lymphocytic leukemia/small lymphocytic lymphoma or mantle cell lymphoma. Clin Lymphoma Myeloma Leuk. 2018;18(10):648-657. e15. doi:10.1016/j.clml.2018.06.016

45. Barf T, Covey T, Izumi R, et al. Acalabrutinib (ACP-196): a covalent Bruton tyrosine kinase inhibitor with a differentiated selectivity and in vivo potency profile. $J$ Pharmacol Exp Ther. 2017;363 (2):240-252. doi:10.1124/jpet.117.242909

46. Wang M, Rule S, Zinzani PL, et al. Acalabrutinib in relapsed or refractory mantle cell lymphoma (ACE-LY-004): a single-arm, multicentre, phase 2 trial. Lancet. 2018;391(10121):659-667. doi:10.1016/ S0140-6736(17)33108-2

47. Wang M, Rule S, Zinzani PL, et al. Durable response with single-agent acalabrutinib in patients with relapsed or refractory mantle cell lymphoma. Leukemia. 2019;33(11):2762-2766. doi:10.1038/s41375-019-0575-9

48. Byrd JC, Owen R, O’Brien SM, et al. Pooled analysis of safety data from clinical trials evaluating acalabrutinib monotherapy in hematologic malignancies. Blood. 2017;130(Supplement 1):4326. doi:10. 1182/blood.V130.Suppl 1.4326.4326

49. Song Y, Zhou K, Zou D, et al. Safety and activity of the investigational bruton tyrosine kinase inhibitor zanubrutinib (BGB-3111) in patients with mantle cell lymphoma from a phase 2 trial. Blood. 2018;132((Supplement 1)):148. doi:10.1182/blood-2018-99-117956

50. Song Y, Zhou K, Zou D, et al. ZANUBRUTINIB IN PATIENTS WITH RELAPSED/REFRACTORY MANTLE CELL LYMPHOMA. Hematol Oncol. 2019;37(S2):45-46. doi:10.1002 hon. $15 \_2629$

51. Tam CS, Wang M, Simpson D, et al. Updated safety and efficacy data in the phase 1 trial of patients with Mantle Cell Lymphoma $(\mathrm{Mcl})$ treated with Bruton Tyrosine Kinase (Btk) inhibitor zanubrutinib (Bgb-3111). Hematol Oncol. 2019;37:245-247. doi:10.1002/ hon.55 2630

52. Tam CS, Opat S, Zhu J, et al. PS1159 pooled analysis of safety data from monotherapy studies of the bruton tyrosine kinase (BTK) inhibitor, zanubrutinib (BGB-3111), in B-cell malignancies. HemaSphere. 2019;3(S1):526. doi:10.1097/01.HS9.0000562920.266 $03.5 \mathrm{~b}$

53. Tam CSL, Opat S, D'Sa S, et al. ASPEN: results of a phase III randomized trial of zanubrutinib versus ibrutinib for patients with Waldenström macroglobulinemia (WM). J Clin Oncol. 2020;38 (15 suppl):8007. doi:10.1200/JCO.2020.38.15 suppl.8007

54. Wang M, Rule S, Zinzani PL, et al. Long-term follow-up of acalabrutinib monotherapy in patients with relapsed/refractory mantle cell lymphoma. Blood. 2018;132(Supplement 1):2876. doi:10.1182/ blood-2018-99-110327
OncoTargets and Therapy

\section{Publish your work in this journal}

OncoTargets and Therapy is an international, peer-reviewed, open access journal focusing on the pathological basis of all cancers, potential targets for therapy and treatment protocols employed to improve the management of cancer patients. The journal also focuses on the impact of management programs and new therapeutic

Submit your manuscript here: https://www.dovepress.com/oncotargets-and-therapy-journ agents and protocols on patient perspectives such as quality of life, adherence and satisfaction. The manuscript management system is completely online and includes a very quick and fair peer-review system, which is all easy to use. Visit http://www.dovepress.com/ testimonials.php to read real quotes from published authors. 